Research Article

\title{
Convective Mass/Heat Analysis of an Electroosmotic Peristaltic Flow of Ionic Liquid in a Symmetric Porous Microchannel with Soret and Dufour
}

\author{
Humaira Yasmin $\mathbb{I D}^{1}$ and Naveed Iqbal $\mathbb{D}^{2}$ \\ ${ }^{1}$ Department of Basic Sciences, Preparatory Year Deanship, King Faisal University, Al-Ahsa 31982, Saudi Arabia \\ ${ }^{2}$ Department of Mathematics, College of Science, University of Ha'il, Ha'il 81481, Saudi Arabia \\ Correspondence should be addressed to Humaira Yasmin; qau2011@gmail.com
}

Received 13 May 2021; Revised 4 June 2021; Accepted 16 June 2021; Published 28 June 2021

Academic Editor: Ahmed Zeeshan

Copyright (c) 2021 Humaira Yasmin and Naveed Iqbal. This is an open access article distributed under the Creative Commons Attribution License, which permits unrestricted use, distribution, and reproduction in any medium, provided the original work is properly cited.

\begin{abstract}
This research deals with the mathematical model for the development of the peristaltic principle of the combination of the pressure and electroosmotic flow (EOF) of ionic liquid across microchannels with electrokinetic effects. For thermomechanical dynamics, the convective conditions on the boundary for mass and heat transfer at the walls of the channel are quantified. For the microchannel, a porous structure is presumed. Soret, Dufour, and Joule heating are also listed in the scope of the problem addressed. The corresponding equations for the ionic fluid flow, mass, and heat transfer along with the Poisson-Boltzmann equation within the electrical double layer (EDL) are studied. The exact solution has been obtained based on lubrication theory (i.e., low Reynolds number and long wavelength approximations). The channel height is therefore believed to be much higher than the electrical double layer (EDL) thickness. Various dimensionless pertinent parameters illustrate the important aspects of electroosmotically controlled flow and subsequent convective mass/heat transfer attributes in a microchannel. A linear dependency on the fluid flow rate is exhibited by the pressure drop. The analysis shows that the electroosmotic parameter gives a reducing effect on the channel permeability. The distribution of temperature and concentration is greatly affected by convective heat and mass parameters, respectively. In biomedical engineering, the application areas of the study proposed are for the design of the devices such as a microfluidic pump to pump a small amount of ionic liquids by regulating the variation in temperature and concentration.
\end{abstract}

\section{Introduction}

Electroosmosis has been seen to a considerable extent in recent decades. Microelectromechanical System (MEMS) was the first device with microfluidic technology assembled in the 1980s. The installations of these devices are widespread in a range of contexts comprising biological and biomedical industry sectors, where microfluidic products are termed as LOC (lab-on-chip devices). These devices can also be used for a variety of purposes like biomedical therapeutics and microbial or toxic contamination. Nowadays, a strong demand is raised on compact, simple, and inexpensive standardized equipment for pharmaceutical dissemination and DNA-based analysis because of the need for effective, rapid, and considerable standardized reliability of microfluidics. Microfluidic devices frequently drive fluid throughout the whole network employing electroosmosis. Electroosmosis represents the electrolyte flow across a channel with an applied voltage charge boundary. In several biological, clinical, and manufacturing systems, electroosmosis develops, including dialysis of fluids, porous membranes, zoological processes, transportation in human skin, tube/canal flow, and techniques of separation. The simulation of EOF was projected by Patankar and $\mathrm{Hu}$ [1]. In capillary assessments, Huang et al. [2] demonstrated the electroosmotic flow (EOF). The composition characteristics of microflows were examined on an EOF basis by Haswell [3]. The applications of microfluidics have been addressed by 
Gravesen et al. [4]. In the annulus of capillary walls, Kang et al. [5] also analyzed the EOF.

Investigations on controlled microchannel flow continued to draw interest, leading to its widespread use in various fields of science, especially genetics, medicine, and medical technology. Studies such as this one have shown, on the one hand, that they are capable of revealing interesting characteristics, especially if such channels reflect a longitudinal wave that travels across the length of walls, in this scenario called the peristalsis. The peristalsis is therefore characterized as a wavelike movement inside flexible tubes triggered and operated by a physiological flexion and extension of adjacent locations, which leads to a movement of the within contents. We also note that since this mechanism is becoming increasingly relevant in many ways, it has recently undergone comprehensive study with an even wider impact. Considering its relevance to various industrial and physiological fields, many observations were taken in the studies [6-8]. Furthermore, several biomedical electronic types of equipment, for example, the cardiovascular device and plasma pumps during dialysis, have found this spontaneous phenomenon. However, after one of Latham's first major investigations [9], several researchers had already been motivated to explore the dynamics of peristaltic motion. At that time, there have been numerical $[6-8,10]$ and experimental [11-13] approaches used, where there have been those who have used analytical $[14,15]$ approach to examine and understand the effects of such oscillatory movement's mechanics and physiology. The way that this type of action takes place is by the squeezed displacement of the walls of the channel and their contents. A good example is seen as food is swallowed via the esophagus. Thus, peristaltic pumps are usually demanded in pharmaceutical companies due to their systemic potential for transportation, thus generating considerable interest in transportation studies as they have been done in [13].

In addition, peristaltic pumping is not infrequent to be subject to other external consequences which can seriously impair it. This mechanism has been extensively investigated and is often used with effects such as heat transfer [8], electroosmosis [16], and magnetic fields [7]. While this type of analysis usually results in nonlinear procedures, a relatively small number has been designed to give rise to linear forms. Consequently, several mathematical models have thus been explored governing peristaltic flow. The potential effects of peristaltic pumping and common external effects (electromagnetic, electroosmotic) [17-23], which have been documented in the literature, influence particularly physiological flows. The narrowness of the peristaltic conduits has made it highly appealing for the lubrication assumption. Despite its success, other researchers have prevented this approach, but they have given the solutions for these dynamics [6-8]. In addition, the studied results alongside peristalsis highlight the non-Newtonian parameters, the thickness of the electrical double layer (abbreviated as EDL), the external electromagnetic field, slip parameter, Joule heating, flow characteristics, and radiation. Surprisingly, there was also an overview of entropy generation analysis [18]. Indeed how this impact alters peristaltic transports significantly is interesting.
In terms of tissue degradation, portable disease testing kits, analysis of dilution strategies in blood flow, and microfabrication technology, heat transfer in microfluidic systems is extremely important. The effect of heat exchange on the third-order fluid in peristaltically driven EOF is investigated by Waheed et al. [24] in EOF. The heat flow impact of the power-law fluid on the microchannel is reported by Babaie et al. [25]. Besides, Chen et al. [26] have studied the thermal exchange of the non-Newtonian suspension in a microchannel. Sinha and Shit [27] were engaged in thermal blood exchange across capillaries. EOF for heat exchange and MHD also was shown by Shit et al. [28]. Also, the tapered walls in the EOF which improve transfer of mass are regarded in medical operations. Therefore, the heat and mass distribution through EDL effects have been represented by Bhatti et al. [29]. Furthermore, the MHD electroosmotic flow of power-law $\mathrm{Al}_{2} \mathrm{O}_{3}$ PVC nanofluid in a channel was discovered by Shehzad et al. [30]. Yadav et al. [31] also analyzed peristaltic propelling with EDL. The influence of heat in the EOF was investigated by Narla et al. [32] through time. Furthermore, the transfer of heat in MHD EOF was also portrayed by Yang et al. [33].

Both mass and heat transfer impacts play a vital role in the distribution of organic compounds in saturated soil, radioactive waste disposal, thermal insulation, increased oil recovery, and so on. In combustion, fermentation, and distillation and many other manufacturing processes, the impact of the mass transfer appeared. In the case of devices like steam engines, nuclear plants, heat storage, and so forth, convective heat transfer via boundary conditions is used. The results of the heat/mass transfer on the fluid triggering peristaltic motion in fully compliant curved canals were investigated, in this regard, by Hina and Hayat [34]. The characteristics of heat flux in peristaltic transportation with/ without compliant walls were studied by Riaz et al. [35], Hayat et al. [36], and Mehmood et al. [37]. In the presence of convective constraints, different non-Newtonian fluid models with/without peristalsis are analyzed in [38-43] and many references therein. Yasmin et al. [44] addressed the impact of convective conditions in the peristalsis of Johnson-Segalman fluid in an asymmetric channel.

With this development, we notice that the solutions may lead to complicated mathematical procedures when the aforesaid nontrivial hypotheses are implemented into the peristaltic microchannels. Analytical solutions have been achieved $[16,45]$ for basic situations in which the leading governing equations are compatible. The rheological effects of fluid were also studied on the electroosmotic flow in a non-Darcy porous medium $[46,47]$. In other cases, the complexity involved the semianalytical approach, which included the use of numerical schemes [48-50] and perturbation theory [51] to find a complete solution to the problem. However, some effective research without the use of perturbation theory [6-8] was observed in other cases.

We realize that no study on convective heat and mass transfer in EOF altered by peristalsis with Soret, Dufour, Joule heating, and porous medium currently exists. This research covers the void. The main objective of this paper is to study an ionic liquid with viscous dissipation in a 
microchannel with a porous structure, impact of convective heat transfer, and convective mass transfer for EOF. The equations governing the fluid velocity, mass, and heat transfer in the EDL are taken with the famous Poisson-Boltzmann equation. Approximation of lubrication and Debye-Hückel linearization are implemented for this research. The exact solution for the governing equations is obtained. Schematic findings are drawn and discussed thoroughly for physical quantities.

\section{Flow Analysis}

Consider a fully developed unsteady ionic liquid flow via a microchannel bounded by two walls with rhythmic contraction, $\bar{y}= \pm \bar{h}(\bar{x}, \bar{t})$, in accordance with an alternating external electric field and the enacted pressure gradient. Due to wave propagation, the constituent equation for deforming of the wall (see Figure 1) takes the form

$$
\bar{h}(\bar{x}, \bar{t})=d-\bar{a} \cos \left(\frac{\pi}{\lambda}(\bar{x}-c \bar{t})\right)
$$

where the axial channel coordinate, time, half-width of the channel, the amplitude of the wave, the wavelength, and the wave velocity are represented by $\bar{x}, \bar{t}, d, \bar{a}, \lambda$, and $c$ respectively. It is presumed that the flow is symmetrical along the channel center $(\bar{y}=0)$, which means that the flow domain in the area $0 \leq \bar{y} \leq \bar{h}$ is studied. At the walls of the channel, the zeta potential is taken as constant. $\vec{E}=$ $-\vec{\nabla} \bar{V}$ produces both an applied external electric field alongside the microchannel axis and the required electroosmotic fluid flow driving force as well as the peristaltic wave-induced flow. The no-slip conditions for velocity have been taken into consideration, whereas convective heat along with convective mass conditions is applied in this analysis.

Because of the existence of electrical double layer (EDL) in the microchannel as defined by the Poisson-Boltzmann equation [52], for a symmetric $(z: z)$ binary electrolyte solution, the electric potential distribution developed in the Cartesian coordinate can be written as

$$
\frac{\partial^{2} \Phi}{\partial x^{2}}+\frac{\partial^{2} \Phi}{\partial y^{2}}=-\frac{\rho_{e}}{\varepsilon \varepsilon_{0}},
$$

in which $\varepsilon$ is the dielectric constant of the medium, $\rho_{e}$ represents the density of the ionic change, and $\varepsilon_{0}$ is the vacuum permittivity which is equal to $8.854 \times 10^{-12} \cdot \mathrm{CV}^{-1} \mathrm{~m}^{-1} \cdot \rho_{e}$ (density of the ionic energy) for a symmetric electrolyte $(z: z)$ is written as

$$
\rho_{e}=e z\left(n^{+}-n^{-}\right)
$$

in which $n^{-}$is the number of anions' density and $n^{+}$is the number of cations' density and these can be given by Boltzmann distribution with the consideration of no overlapped EDL:

$$
n^{ \pm}=n_{0} \exp \left(\mp \frac{e z \bar{\Phi}}{\kappa_{\mathrm{B}} T_{\mathrm{av}}}\right)
$$

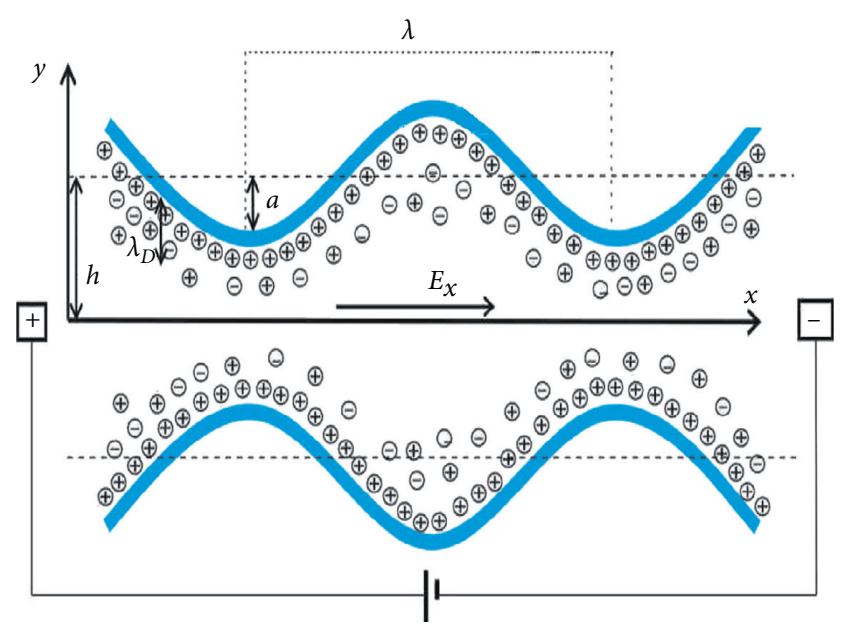

Figure 1: Schematic diagram of the problem.

where $n_{0}$ is the bulk ions concentration, which is independent of electrochemistry surface, $\kappa_{B}$ is the Boltzmann constant, $e$ represents the electric charge, and $T_{\mathrm{av}}$ denotes the average electrolytic solution temperature.

This distribution of the ionic concentration tends to be true if the ionic concentration in the microchannel is not axial and, for fluid flow, the substantially small Peclet number is supposed. From equations (2)-(4), one can get the Poisson-Boltzmann equation as follows:

$$
\frac{\mathrm{d}^{2} \bar{\Phi}}{\mathrm{d} y^{2}}=\frac{2 n_{0} e z}{\varepsilon \varepsilon_{0}} \sinh \left(\frac{e z \bar{\Phi}}{\kappa_{\mathrm{B}} T_{\mathrm{av}}}\right) .
$$

Introduce now a standardized electroosmotic potential function $\bar{\Phi}$ with medium potential $\zeta$ and other dimensionless variables:

$$
\begin{aligned}
\Phi & =\frac{\bar{\Phi}}{\bar{\zeta}}, \\
y & =\frac{\bar{y}}{d}, \\
x & =\frac{\bar{x}}{\lambda}, \\
t & =\frac{c \bar{t}}{\lambda}, \\
h(x, t) & =\frac{h(\bar{x}, \bar{t})}{d}, \\
a & =\frac{\bar{a}}{\bar{d}} .
\end{aligned}
$$

Equation (5) is now read in view of the nondimensionally defined variables from equation (6) as

$$
\frac{\mathrm{d}^{2} \Phi}{\mathrm{d} y^{2}}=\frac{\mathrm{d}^{2}}{\lambda_{D}^{2} \alpha} \sinh (\alpha \Phi),
$$

in which the ionic energy parameter is defined as $\alpha=\left(e z \zeta / \kappa_{B} T_{\mathrm{av}}\right)$ and the Debye length is denoted by 
$\lambda_{D}=(e z)^{-1}\left(\varepsilon \varepsilon_{0} \kappa_{B} T_{a v} / 2 n_{0}\right)^{(1 / 2)}$. As the potential function is symmetrical, the following condition is taken into account:

$$
\frac{\mathrm{d} \Phi}{\mathrm{d} y}=0, \quad \text { at } y=0,
$$

and the potential function $\Phi$ at the borderline should be

$$
\Phi=1, \quad \text { at } y=h .
$$

Further, it is assumed that the wall zeta potential $(\zeta \leq 25 \mathrm{mV})$ is insufficiently small to enable the approximation of the Debye-Hückel linearization.
The linearized Poisson-Boltzmann equation (7) $(\sinh (\alpha \Phi) \approx \alpha \Phi)$ can be resolved in order to achieve the potential distribution by using the boundary conditions (8) and (9) as

$$
\Phi=\frac{\cosh (m y)}{\cosh (m h)},
$$

where the electroosmotic parameter is represented by $m=\left(d / \lambda_{D}\right)$. The study of the presented model is conducted under the impact of all the above assumptions; the biofluid flow equations and the heat transfer via permeable microchannel are considered as $[52,53]$

$$
\begin{aligned}
\frac{\partial \bar{u}}{\partial \bar{x}}+\frac{\partial \bar{v}}{\partial \bar{y}} & =0 \\
\rho\left(\frac{\partial \bar{u}}{\partial t}+\bar{u} \frac{\partial \bar{u}}{\partial x}+\bar{v} \frac{\partial \bar{u}}{\partial \bar{y}}\right) & =-\frac{\partial \bar{p}}{\partial \bar{x}}+\mu\left(\frac{\partial^{2} \bar{u}}{\partial \bar{y}^{2}}+\frac{\partial^{2} \bar{u}}{\partial \bar{x}^{2}}\right)-\mu \frac{\bar{u}}{\bar{k}}+\rho_{e} E_{x}, \\
\rho\left(\frac{\partial \bar{v}}{\partial \bar{t}}+\bar{u} \frac{\partial \bar{v}}{\partial \bar{x}}+\bar{v} \frac{\partial \bar{v}}{\partial \bar{y}}\right) & =-\frac{\partial \bar{p}}{\partial \bar{x}}+\mu\left(\frac{\partial^{2} \bar{v}}{\partial \bar{y}^{2}}+\frac{\partial^{2} \bar{v}}{\partial \bar{x}^{2}}\right)-\mu \frac{\bar{v}}{\bar{k}} \\
\rho c_{p}\left(\frac{\partial T}{\partial \bar{t}}+\bar{u} \frac{\partial T}{\partial \bar{x}}+\bar{v} \frac{\partial T}{\partial \bar{y}}\right) & =k_{1}\left(\frac{\partial^{2} T}{\partial \bar{y}^{2}}+\frac{\partial^{2} T}{\partial \bar{x}^{2}}\right)+\sigma E_{x}^{2}+\mu\left(\frac{\partial \bar{u}}{\partial \bar{y}}\right)^{2}+\frac{D k_{T}}{c_{s}}\left(\frac{\partial^{2} C}{\partial \bar{y}^{2}}+\frac{\partial^{2} C}{\partial \bar{x}^{2}}\right), \\
\rho\left(\frac{\partial C}{\partial \bar{t}}+\bar{u} \frac{\partial C}{\partial \bar{x}}+\bar{v} \frac{\partial C}{\partial \bar{y}}\right) & =D\left(\frac{\partial^{2} C}{\partial \bar{y}^{2}}+\frac{\partial^{2} C}{\partial \bar{x}^{2}}\right)+\frac{D k_{T}}{T_{m}}\left(\frac{\partial^{2} T}{\partial \bar{y}^{2}}+\frac{\partial^{2} T}{\partial \bar{x}^{2}}\right),
\end{aligned}
$$

where the velocity components along $x$-axis and $y$-axis are denoted as $\bar{u}$ and $\bar{v}$, respectively, the fluid density is indicated by $\rho, \mu$ is the viscosity, $\bar{p}$ is the pressure, $c_{p}$ is the specific heat with constant pressure, $T$ is the ionic fluid temperature, with the thermal conductivity being $k_{1}$, the permeability of the medium is $k, E_{x}$ is the electrical field in the $x$ direction, $\sigma$ is the electrical conductivity, $C$ is the fluid concentration, $k_{T}$ is thermal diffusion ratio, $D$ is the coefficient of concentration diffusivity, $c_{s}$ is the susceptibility of concentration, and $T_{m}$ is the mean temperature. The first term on the left-hand side of each equation from (12) to (14) shows the transient state. The terms $\mu(\bar{v} / k)$ and $\mu(\bar{u} / k)$ on the right-hand side of equations (12) and (13) show the permeability of the microchannel, whereas the first term on the right-hand side of equations (12) and (13) represents the applied pressure gradient. The term $\sigma E_{x}^{2}$ of equation (14) provides the effects of Joule heating. In this study, the Joule heating with viscous dissipation in a microchannel is considered. The last term in equation (14) represents the Soret effects, whereas the last term in equation (15) represents the Dufour effects.
The conditions for the particular problem are defined as

$$
\begin{aligned}
\frac{\partial \bar{u}}{\partial \bar{y}} & =0, \\
\frac{\partial T}{\partial \bar{y}} & =0, \\
\frac{\partial C}{\partial \bar{y}} & =0, \quad \text { at } \bar{y} \\
\bar{u} & =\bar{c}, \\
k_{T} \frac{\partial T}{\partial y} & =-h_{t}\left(T-T_{a}\right), \\
D \frac{\partial C}{\partial \bar{y}} & =-h_{m}\left(C-C_{a}\right), \quad \text { at } \bar{y}=h,
\end{aligned}
$$

where $h_{t}$ and $h_{m}$ represent the convective heat and mass transfer coefficients, respectively, $T_{a}$ is the ambient temperature at the walls, and $C_{a}$ is the ambient concentration at 
the walls. The conditions on heat and mass transfer are called convective boundary conditions. The source of these boundary conditions is given in $[40,41,44]$ and many references therein.

Let us now establish the following dimensionless variable quantities:

$$
\begin{aligned}
& p=\frac{\bar{p} d^{2}}{\mu c \lambda}, \\
& \operatorname{Re}=\frac{\rho c d}{\mu}, \\
& \delta=\frac{d}{\lambda}, \\
& k=\frac{\bar{k}}{d^{2}}, \\
& \theta=\frac{T-T_{a}}{T_{a}}, \\
& \varphi=\frac{C-C_{a}}{C_{a}}, \\
& \operatorname{Pr}=\frac{\mu c_{p}}{k_{1}}, \\
& u=\frac{\bar{u}}{c}, \\
& v=\frac{\bar{v}}{c \delta}, \\
& \gamma=\frac{\sigma E_{x}^{2} d^{2}}{k_{T} T_{a}}, \\
& \mathrm{Br}=\frac{\mu c^{2}}{k_{T} T_{a}}, \\
& \mathrm{Du}=\frac{D c_{a}}{c_{s} T_{a}}, \\
& \mathrm{Sc}=\frac{\mu}{D \rho} \text {, } \\
& \mathrm{Sr}=\frac{D k_{T} T_{a} \rho}{\mu T_{m} C_{a}}, \\
& \mathrm{Bi}_{t}=\frac{h_{t} d}{k_{T}}, \\
& \mathrm{Bi}_{m}=\frac{h_{m} d}{D},
\end{aligned}
$$

where $p$ is the dimensionless pressure, Re is the Reynolds number, $\delta$ is the wave number, $k$ is the permeability parameter, $\operatorname{Pr}$ is the Prandtl number, $\gamma$ is defined as the Joule heating parameter, $\mathrm{Br}$ is the Brinkman number, $\mathrm{Du}, \mathrm{Sc}$, and $\mathrm{Sr}$ are the Dufour, Schmidt, and Soret numbers, respectively,

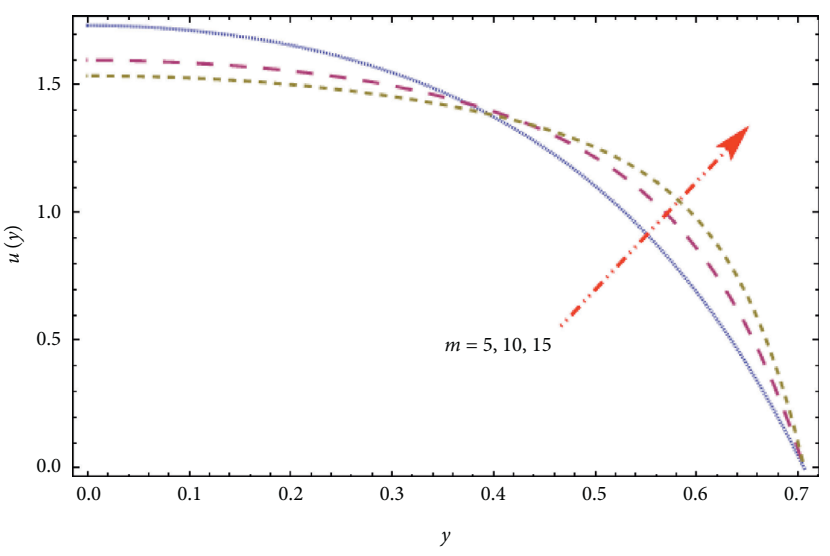

Figure 2: Graph of $u(y)$ versus $m$.

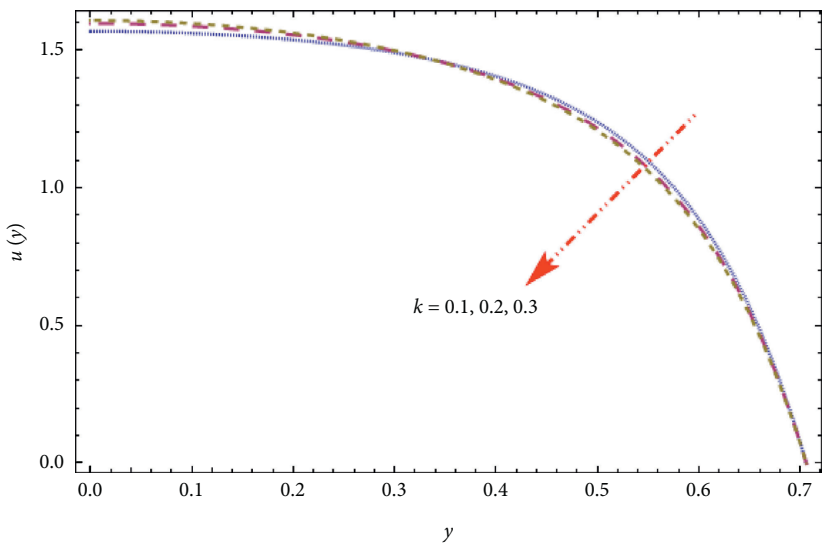

FIGURE 3: Graph of $u(y)$ versus $k$.

and $\mathrm{Bi}_{t}$ and $\mathrm{Bi}_{m}$ indicate the Biot numbers for heat and mass transfer, respectively. Introducing these dimensionless variables into equations (11)-(15), the nondimensional governing equations may be put as

$$
\begin{aligned}
& \frac{\partial u}{\partial x}+\frac{\partial u}{\partial y}=0, \\
& \operatorname{Re} \delta^{2}\left(\frac{\partial u}{\partial t}+u \frac{\partial u}{\partial x}+v \frac{\partial u}{\partial y}\right)=-\frac{\partial p}{\partial x}+\left(\delta^{2} \frac{\partial^{2} u}{\partial x^{2}}+\frac{\partial^{2} u}{\partial y}\right)-\frac{u}{k}+\beta m^{2} \Phi, \\
& \operatorname{Re} \delta^{2}\left(\frac{\partial v}{\partial t}+u \frac{\partial v}{\partial x}+v \frac{\partial v}{\partial y}\right)=-\frac{\partial p}{\partial y}+\left(\delta^{2} \frac{\partial^{2} v}{\partial x^{2}}+\frac{\partial^{2} v}{\partial y^{2}}\right)-\delta^{2} \frac{v}{k}, \\
& \operatorname{Re} \delta \operatorname{Pr}\left(\frac{\partial \theta}{\partial t}+u \frac{\partial \theta}{\partial x}+v \frac{\partial \theta}{\partial y}\right)=\left(\delta^{2} \frac{\partial^{2} \theta}{\partial x^{2}}+\frac{\partial^{2} \theta}{\partial y^{2}}\right)+\gamma+\operatorname{Br}\left(\frac{\partial u}{\partial y}\right)^{2} \\
& +\operatorname{Du}\left(\delta^{2} \frac{\partial^{2} \varphi}{\partial x^{2}}+\frac{\partial^{2} \varphi}{\partial y^{2}}\right)
\end{aligned}
$$




$$
\begin{aligned}
\operatorname{Re} \delta\left(\frac{\partial \varphi}{\partial t}+u \frac{\partial \varphi}{\partial x}+v \frac{\partial \varphi}{\partial y}\right)= & \frac{1}{\operatorname{Sc}}\left(\delta^{2} \frac{\partial^{2} \varphi}{\partial x^{2}}+\frac{\partial^{2} \varphi}{\partial y^{2}}\right) \\
& +\operatorname{Sr}\left(\delta^{2} \frac{\partial^{2} \theta}{\partial x^{2}}+\frac{\partial^{2} \theta}{\partial y^{2}}\right)
\end{aligned}
$$

where $\beta=\left(U_{\mathrm{HS}} / c\right)$ denotes the medium mobility and $U_{\mathrm{HS}}$ represents the maximum electroosmotic or Helmholtz-Smoluchowski velocity defined by $U_{\mathrm{HS}}=\left(-E_{x} \varepsilon \varepsilon_{0} \zeta / \mu\right)$. By following the approximations of long wavelength and low Reynolds number, equations (18)-(22) correspond to

$$
\begin{aligned}
& 0=-\frac{\partial p}{\partial x}+\frac{\partial^{2} u}{\partial y^{2}}-\frac{u}{k}+\beta m^{2} \Phi, \\
& 0=-\frac{\partial p}{\partial y}, \\
& 0=\frac{\partial^{2} \theta}{\partial y^{2}}+\gamma+\operatorname{Br}\left(\frac{\partial u}{\partial y}\right)^{2}+\operatorname{Du} \frac{\partial^{2} \varphi}{\partial y^{2}}, \\
& 0=\frac{1}{\operatorname{Sc}} \frac{\partial^{2} \varphi}{\partial y^{2}}+\operatorname{Sr} \frac{\partial^{2} \theta}{\partial y^{2}} .
\end{aligned}
$$

In view of the problem's physical conditions, the constraints can be mathematically expressed as

$$
\begin{aligned}
\frac{\partial u}{\partial y} & =0, \\
\frac{\partial \theta}{\partial y} & =0, \\
\frac{\partial \phi}{\partial y} & =0, \quad \text { at } y=0, \\
u & =0, \\
\frac{\partial \theta}{\partial y} & =-\mathrm{Bi}_{t} \theta, \\
\frac{\partial \varphi}{\partial y} & =-\mathrm{Bi}_{m} \varphi, \quad \text { at } y=h=1-a \cos (\pi(x-t)) .
\end{aligned}
$$

Let us introduce the stream function $\psi$, given by $u=$ $(\partial \psi / \partial y)$ and $v=-(\partial \psi / \partial x)$, which satisfies the continuity equation (18) automatically. Equation (19) can be written as follows:

$$
\frac{\partial p}{\partial x}=\frac{\partial^{3} \psi}{\partial y^{3}}-\frac{1}{k} \frac{\partial \psi}{\partial y}+\frac{\beta m^{2}}{\cosh (m h)} \cosh (m y) .
$$

After eliminating the pressure term, one can obtain

$$
\frac{\partial^{4} \psi}{\partial y^{4}}-\frac{1}{k} \frac{\partial^{2} \psi}{\partial y^{2}}+\frac{\beta m^{3}}{\cosh (m h)} \sinh (m y)=0
$$

The boundary conditions in terms of stream function can be written as

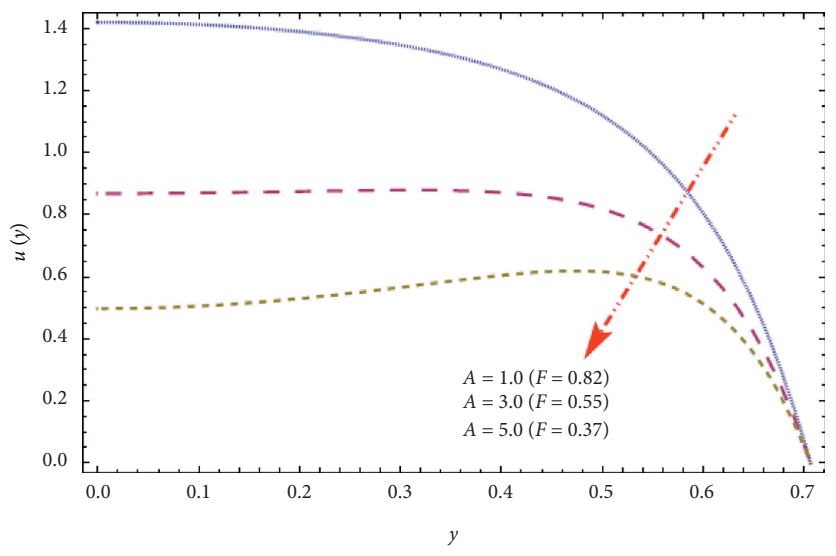

FIGURE 4: Graph of $u(y)$ versus $F$.

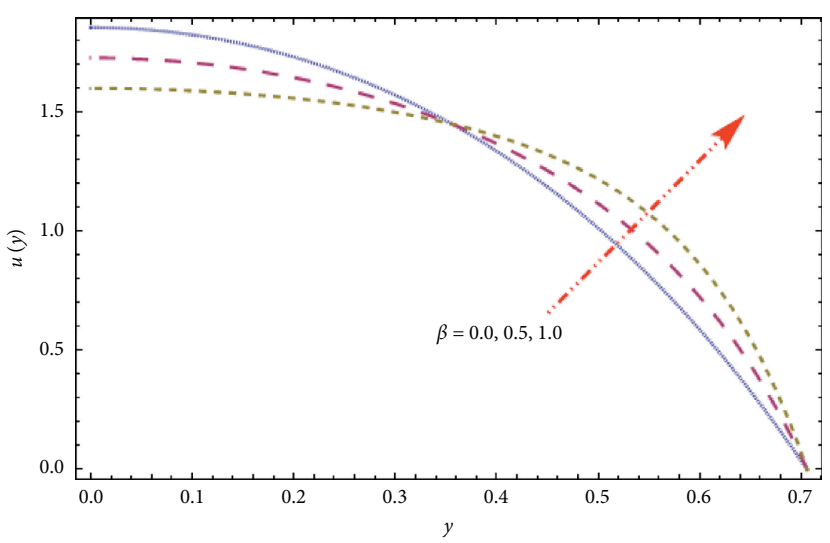

FIgURE 5: Graph of $u(y)$ versus $\beta$.

$$
\begin{aligned}
\psi & =0, \\
\frac{\partial^{2} \psi}{\partial y^{2}} & =0, \\
\frac{\partial \theta}{\partial y} & =0, \\
\frac{\partial \varphi}{\partial y} & =0, \quad \text { at } y=0, \\
\psi & =F, \\
\frac{\partial \psi}{\partial y} & =0, \\
\frac{\partial \theta}{\partial y} & =-\mathrm{Bi}_{t} \theta, \\
\frac{\partial \varphi}{\partial y} & =-\mathrm{Bi}_{m} \varphi, \quad \text { at } y=h=1-a \cos (\pi(x-t)) .
\end{aligned}
$$

It is worth mentioning here that, for obtaining the solution of ODE in $\psi$ as it exists in equation (26) after removal of the pressure term, two additional boundary conditions are 
placed on the stream function. Dimensionless flow rate $F$ is supposed to vary exponentially with constant $A$ and $Q_{0}$ given by $F=Q_{0} e^{-A t}$. Constant $Q_{0}$ is accountable for the negative and positive flow rate $F<0$ or $F>0$ according to $Q_{0}<0$ or $Q_{0}>0$, respectively. When the flow is contrary to the peristaltic movement direction, the negative flow rate reveals retrograde or reverse pumping. The positive pumping is, however, the case where both pressure rise and volumetric flow rate are positive. The blood flow rate reduces exponentially over time, experimentally demonstrated by Kikuchi [54]. This variance in flow rates was also proposed to be not dependent on the microchannel's structural information.

\section{Analytical Solutions}

Solving the system of equations subject to the relevant boundary conditions yields the following solutions for stream function and temperature. The following solutions exist for stream function, temperature, and concentration profiles for the system according to the required boundary conditions:

$$
\begin{aligned}
& \psi(y)=a_{1} \sinh (m y)+a_{2} \sinh \left(\frac{y}{\sqrt{k}}\right)+a_{3} y, \\
& u(y)=\frac{\partial \psi}{\partial y}=m a_{1} \cosh (m y)+\frac{a_{2}}{\sqrt{k}} \cosh \left(\frac{y}{\sqrt{k}}\right)+a_{3}, \\
& \theta(y)=a_{7}\left[2 h\left(2+\mathrm{Bi}_{t} h\right) a_{5}^{2} a_{6}-2 \mathrm{Bi}_{t} a_{5}^{2} y^{2} a_{6}+a_{2}^{2} \mathrm{Bi}_{t} k a_{5}^{2}\left(-\cosh \left(\frac{2 h}{\sqrt{k}}\right)+\cosh \left(\frac{2 y}{\sqrt{k}}\right)\right)\right] \\
& +a_{1}^{2} \mathrm{Bi}_{t} \mathrm{Br}^{2} m^{2} a_{5}^{2}(-\cosh (2 m h)+\cosh (2 m y))-32 a_{1} a_{2} \mathrm{Bi}_{t} \operatorname{Br} k^{5 / 2} m^{3} \cosh \left(\frac{y}{\sqrt{k}}\right) \cosh (m y) \\
& +16 a_{1} a_{2} \operatorname{Br} k^{2} m^{2} \sinh \left(\frac{h}{\sqrt{k}}\right)\left(-m a_{5} \cosh (m h)-\mathrm{Bi}_{t} a_{4} \sinh (m h)\right)-2 a_{2}^{2} \operatorname{Br} \sqrt{k} a_{5}^{2} \sinh \left(\frac{2 h}{\sqrt{k}}\right) \\
& 16 a_{1} a_{2} \operatorname{Br} k^{3 / 2} m^{2} \cosh \left(\frac{h}{\sqrt{k}}\right)\left(2 \mathrm{Bi}_{t} k m \cosh (m h)+a_{5} \sinh (m h)\right)-2 a_{1}^{2} \mathrm{Br}^{2} m^{3} a_{4} \sinh (2 m h) \\
& \left.+16 a_{1} a_{2} \operatorname{Bi}_{t} \operatorname{Br} k^{2} m^{2} a_{4} \sinh \left(\frac{y}{\sqrt{k}}\right) \sinh (m y)\right] \text {, } \\
& \varphi(y)=a_{8} \operatorname{ScSr}\left[-2 h\left(2+\mathrm{Bi}_{m} h\right) a_{5}^{2} a_{6}+2 \mathrm{Bi}_{m} a_{5}^{2} y^{2} a_{6}+a_{2}^{2} \operatorname{Bi}_{m} \operatorname{Br} k a_{5}^{2}\left(\cosh \left(\frac{2 h}{\sqrt{k}}\right)-\cosh \left(\frac{2 y}{\sqrt{k}}\right)\right)\right] \\
& +a_{1}^{2} \mathrm{Bi}_{t} \operatorname{Brk}^{2} m^{2} a_{5}^{2}(-\cosh (2 m h)+\cosh (2 m y))-32 a_{1} a_{2} \operatorname{Bi}_{t} \operatorname{Br} k^{5 / 2} m^{3} \cosh \left(\frac{y}{\sqrt{k}}\right) \cosh (m y) \\
& +16 a_{1} a_{2} \operatorname{Br} k^{2} m^{2} \sinh \left(\frac{h}{\sqrt{k}}\right)\left(-m a_{5} \cosh (m h)-\mathrm{Bi}_{t} a_{4} \sinh (m h)\right)-2 a_{2}^{2} \operatorname{Br} \sqrt{k} a_{5}^{2} \sinh \left(\frac{2 h}{\sqrt{k}}\right) \\
& -16 a_{1} a_{2} \mathrm{Br} k^{3 / 2} m^{2} \cosh \left(\frac{h}{\sqrt{k}}\right)\left(2 \mathrm{Bi}_{m} k m \cosh (m h)+a_{5} \sinh (m h)\right)+2 a_{1}^{2} \mathrm{Br} k^{2} m^{3} a_{5}^{2} \sinh (2 m h) \\
& \left.-16 a_{1} a_{2} \operatorname{Bi}_{m} \operatorname{Br} k^{2} m^{2} a_{4} \sinh \left(\frac{y}{\sqrt{k}}\right) \sinh (m y)\right]
\end{aligned}
$$




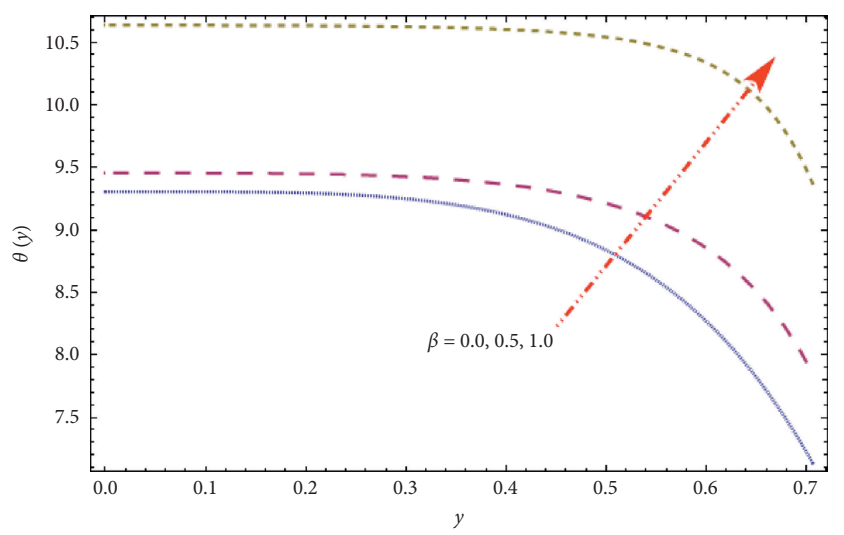

Figure 6: Graph of $\theta(y)$ versus $\beta$.

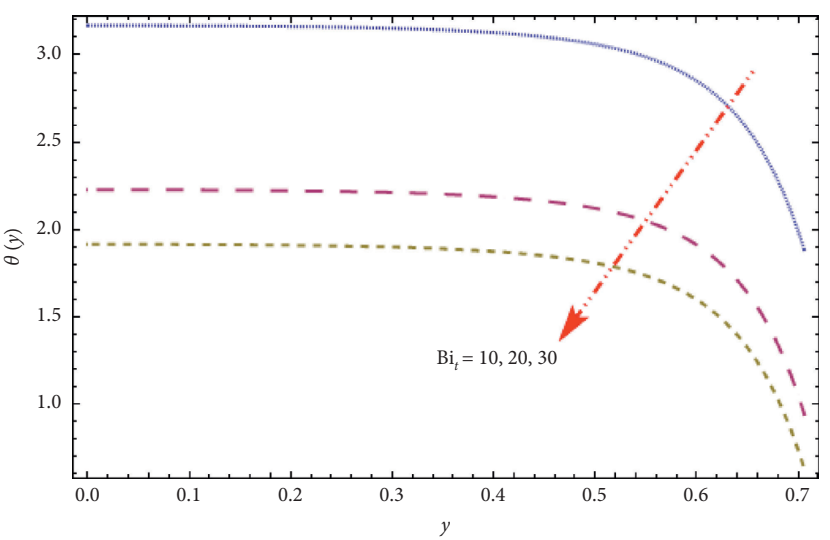

FiguRe 7: Graph of $\theta(y)$ versus $\mathrm{Bi}_{t}$.

where

$$
\begin{aligned}
& a_{1}=a_{9}\left[2 e^{h / \sqrt{k}} \operatorname{sech}(m h)\left(-h k m \beta \cosh \left(\frac{h}{\sqrt{k}}\right)+k^{3 / 2} m \beta \sinh \left(\frac{h}{\sqrt{k}}\right)\right)\right], \\
& a_{2}=a_{9}\left[2 e^{h / \sqrt{k}} \sqrt{k}\left(F-F k m^{2}+h k m^{2} \beta-k m \beta \tanh (m h)\right)\right], \\
& a_{3}=a_{9}\left[2 e^{h / \sqrt{k}}\left(F a_{5} \cosh \left(\frac{h}{\sqrt{k}}\right)-k^{3 / 2} m^{2} \beta \sinh \left(\frac{h}{\sqrt{k}}\right)+k m \beta \cosh \left(\frac{h}{\sqrt{k}}\right) \tanh (m h)\right)\right], \\
& a_{4}=1+k m^{2}, \\
& a_{5}=-1+k m^{2}, \\
& a_{6}=a_{2}^{2} \mathrm{Br}^{2} k^{2}\left(a_{1}^{2} \mathrm{Br} m^{4}-2 \gamma\right), \\
& a_{7}=\frac{1}{8 \mathrm{Bi}_{t} k^{2} a_{5}^{2} a_{10}}, \\
& a_{8}=\frac{1}{8 \mathrm{Bi}_{m} k^{2} a_{5}^{2} a_{10}}, \\
& a_{10}=-1+\operatorname{DuSc}_{5}\left(h+e^{2 h / \sqrt{k}}(h-\sqrt{k})+\sqrt{k}\right),
\end{aligned}
$$

The results presented in the section are in good agreement with the previous study by Ranjit et al. [17] in the absence of convective heat/mass transfer conditions.

\section{Results and Discussion}

Analytical solutions have been acquired and reported in the previous sections on the axial velocity, stream function, pressure gradient, temperature, and concentration characteristics. We provided a series of figures in this section, describing the effects of different parameters of interest on flow quantities such as the axial velocity $u(y)$, distribution of temperature $\theta(y)$, and concentration $\varphi(y)$ by setting the parametric values $a=0.5, \quad t=0.2, \quad x=0.5, \quad Q_{0}=1$,
$A \in[1,5], k \in[0.1,1], m \in[0,20], \beta \in[0,1], \mathrm{Bi}_{t} \in(0,30]$, $\mathrm{Sr} \in[0,1], \mathrm{Du} \in[0,4], \mathrm{Sc} \in[0,1], \mathrm{Br} \in[0,2], \gamma \in[0,1]$, and $\mathrm{Bi}_{m} \in(0,30]$. Figures 2-4 demonstrate a change in axial velocity for several parameters. The consequences on the axial velocity of said electroosmotic parameter $m$ can be seen in Figure 2. It reveals that the velocity profile depends highly on $m$ through the channel. The axial fluid velocity is increased when the values of the electroosmotic parameter $m$ rise in the neighborhood of the channel's wall. Because $m$ is the ratio between channel height $y=h$ and the Debye thickness $\lambda_{D}$, an increasing trend in channel height results in an increase of the axial fluid velocity. The influence of permeability $k$ on fluid velocity is illustrated in Figure 3 . This graph represents that a boost in the value of the permeability 


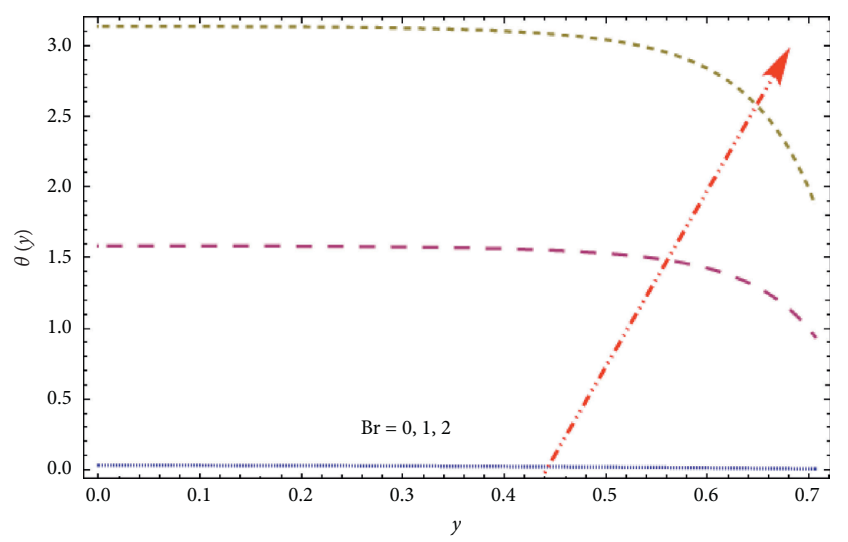

Figure 8: Graph of $\theta(y)$ versus Br.

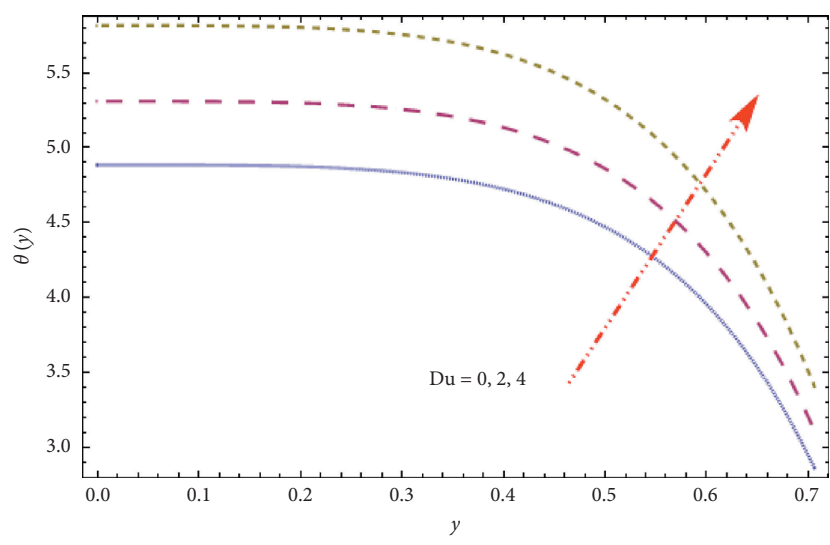

Figure 9: Graph of $\theta(y)$ versus Du.

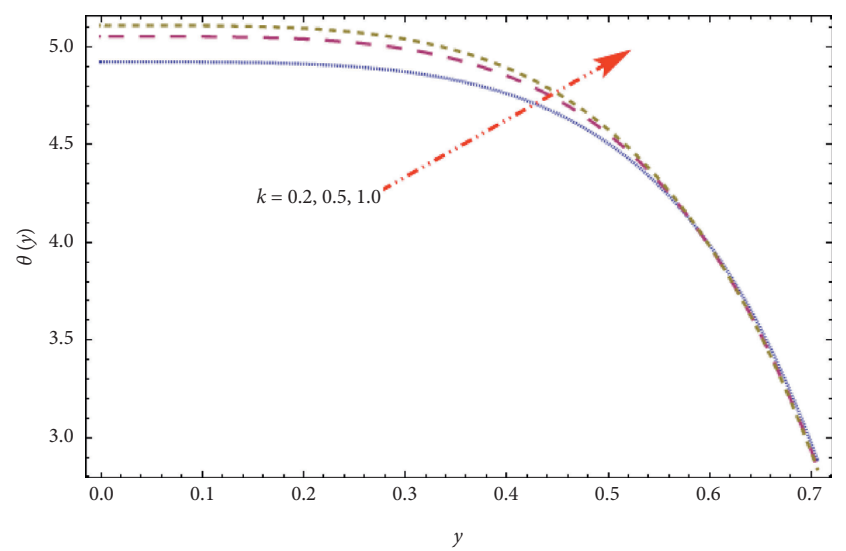

FIgURE 10: Graph of $\theta(y)$ versus $k$.

parameter $k$ raises the fluid velocity in the middle of the channel, while the fluid speed decreases across the walls of the channel. From Figure 4, we noted that, with decreasing flow rate $F$, the axial velocity tends to decrease. We noted that the reduced volumetric flow rate $F$ indicates that a phenomenon called reflux flow occurs in reverse flow. A reflux flow (that occurs for the critical flow rate) corresponding to the preference of the physical parameters is therefore very straightforward to quantify. Figure 5 highlights the results of the fluid velocity profile for the mobility

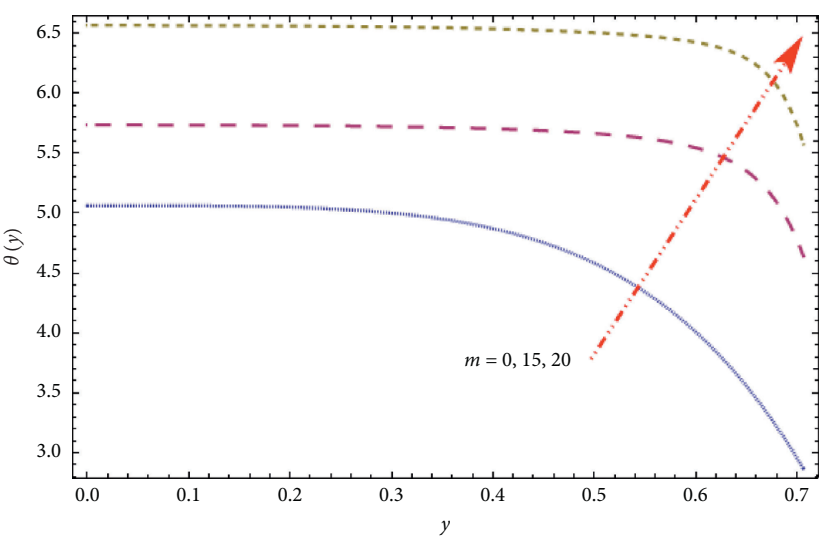

FIGURE 11: Graph of $\theta(y)$ versus $m$.

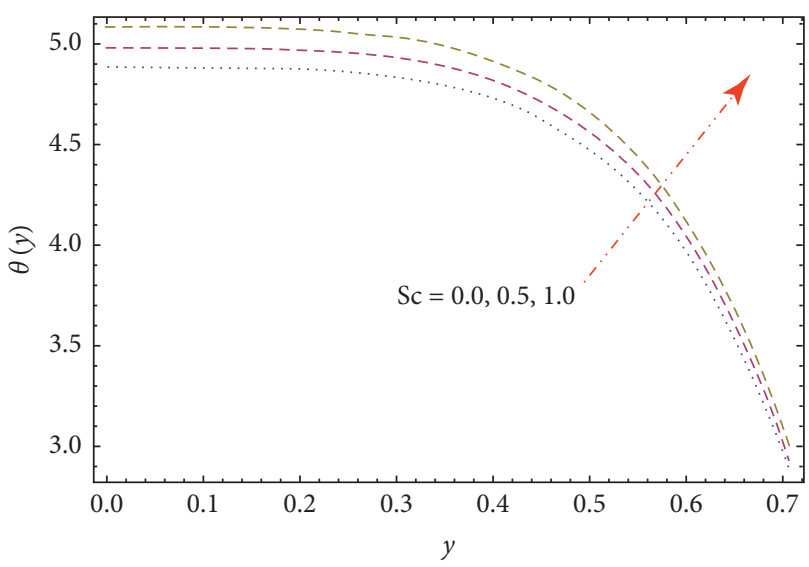

FIgURE 12: Graph of $\theta(y)$ versus Sc.

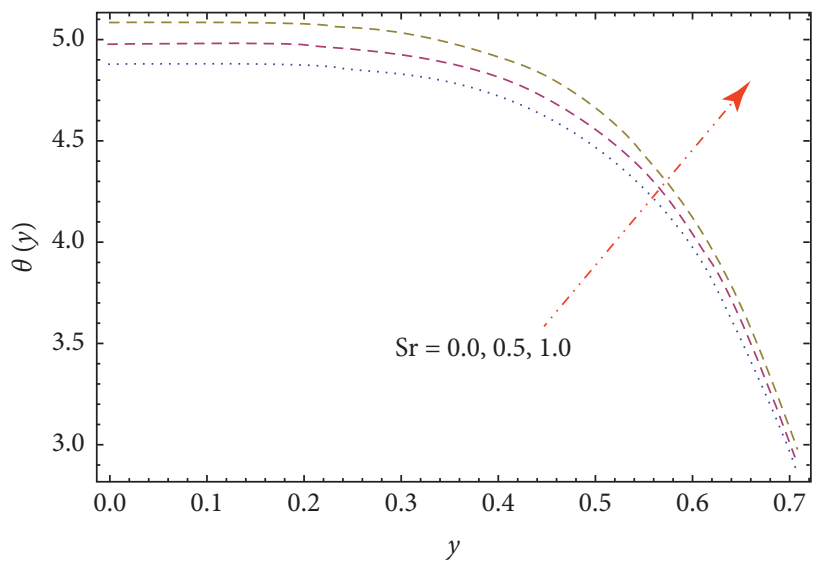

Figure 13: Graph of $\theta(y)$ versus Sr.

of the porous medium $\beta$. It indicates that the axial velocity lowers when the value of $\beta$ grows. As $\beta$ depends directly on Helmholtz-Smoluchowski velocity $U_{H S}$, this can be perceived physically as follows: with increased EDL thickness, the fluid's velocity declines, and the fluid flow decreases when EDL is present. In Figures 6-14, the impact on the fluid temperature of the relevant parameters is shown. Figure 6 shows the influence of the mobility of the medium $\beta$. It reveals that the temperature of the liquid decreases with the 


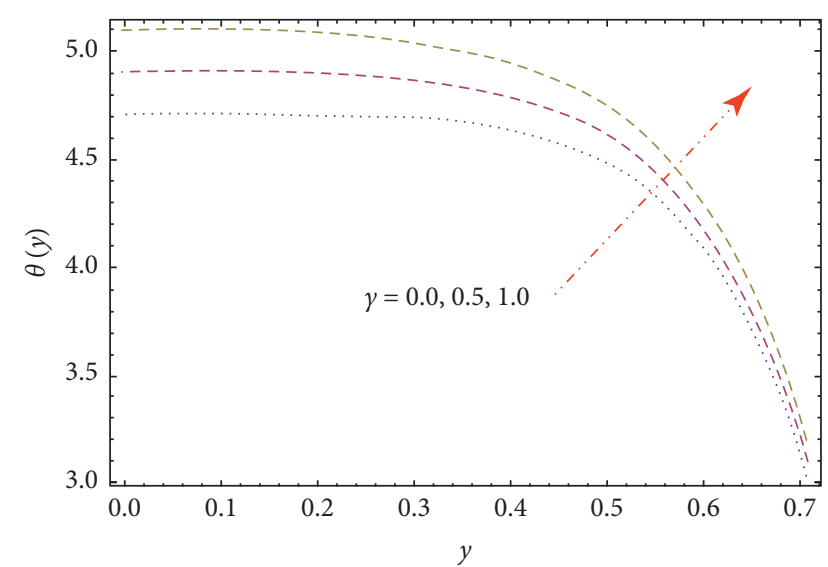

Figure 14: Graph of $\theta(y)$ versus $\gamma$.

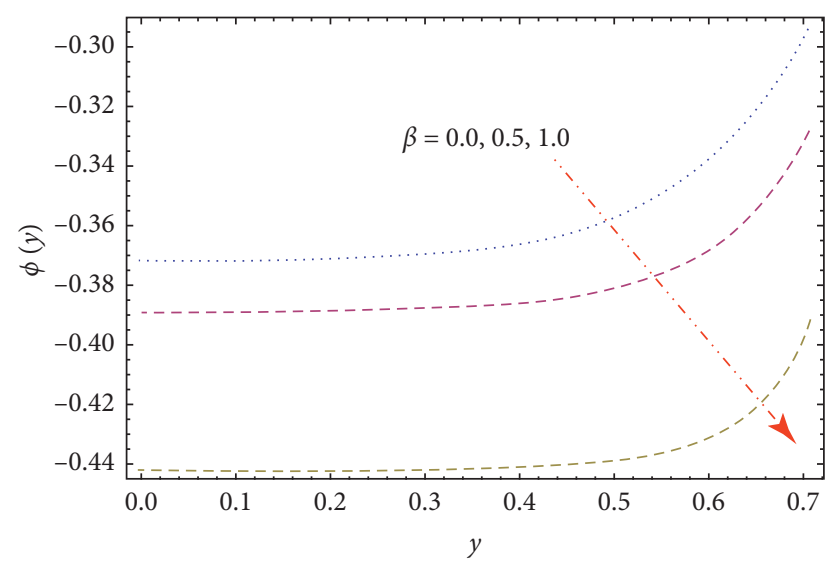

Figure 15: Graph of $\varphi(y)$ versus $\beta$.

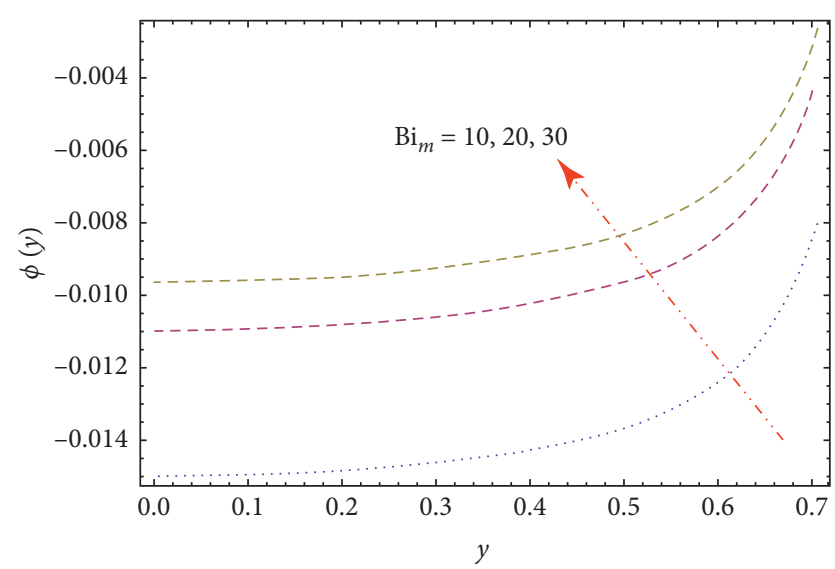

FIgURE 16: Graph of $\varphi(y)$ versus $\mathrm{Bi}_{m}$.

greater mobility of the medium. As $\beta$ depends directly on Helmholtz-Smoluchowski velocity $U_{\mathrm{HS}}$, this can be perceived physically as follows: with increased EDL thickness, the fluid's velocity declines, and the fluid flow decreases when EDL is present; hence, it decreases the fluid temperature. To study the effect of thermal Biot number $\mathrm{Bi}_{t}$ on fluid temperature, Figure 7 is portrayed. It can be seen that the fluid temperature decays as the value of the thermal Biot

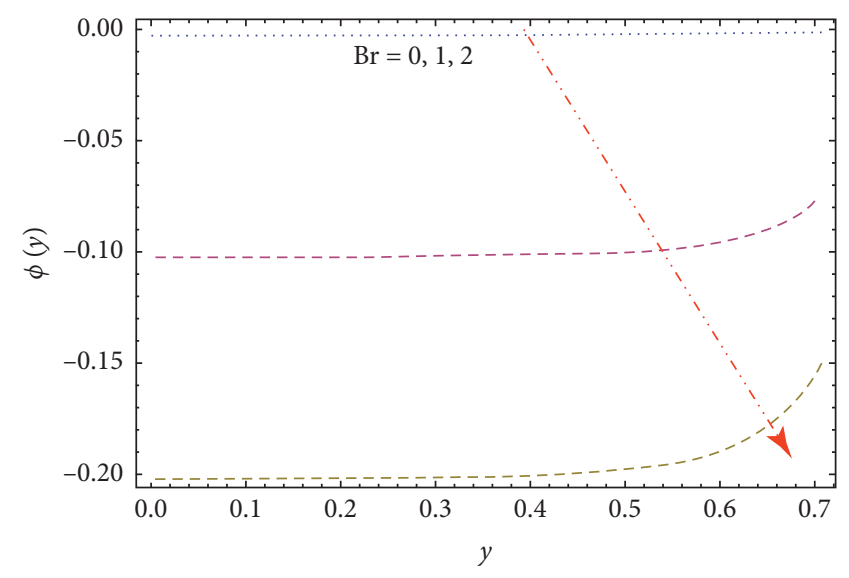

Figure 17: Graph of $\varphi(y)$ versus Br.

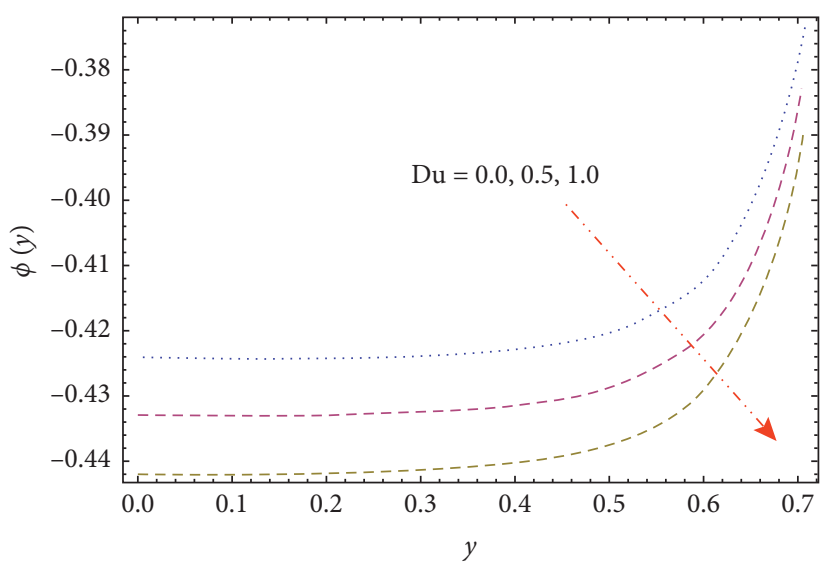

Figure 18: Graph of $\varphi(y)$ versus Du.

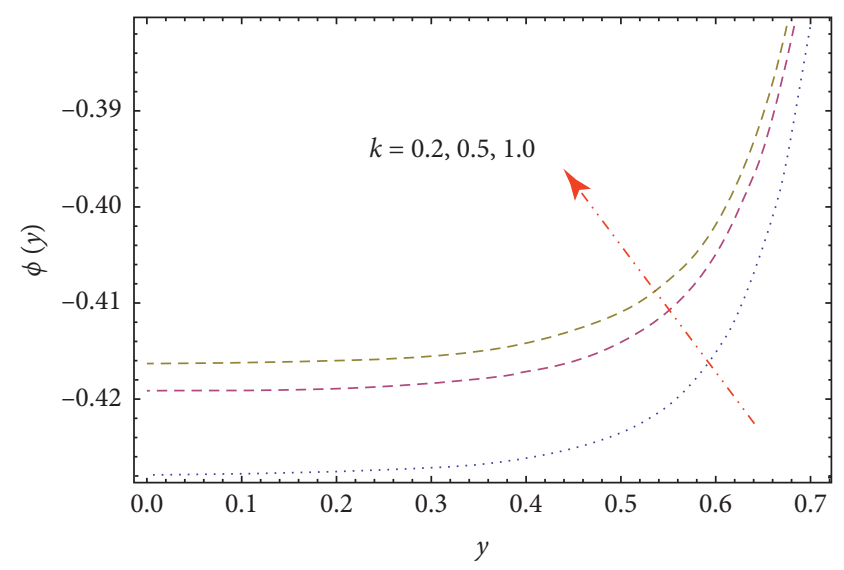

Figure 19: Graph of $\varphi(y)$ versus $k$.

number is increased. Figure 8 depicts the effect of the Brinkman number $\mathrm{Br}$ on the temperature profile. The fluid temperature rises when the Brinkman number is starting to rise. The principal truth in $\mathrm{Br}$ is the resistance creating viscosity. This resistance induces a collision between fluid particles, which causes a rise in temperature in the resulting collision. Increased fluid temperature is observed in Figure 9 due to a rise in the value of the Dufour number Du. For the 


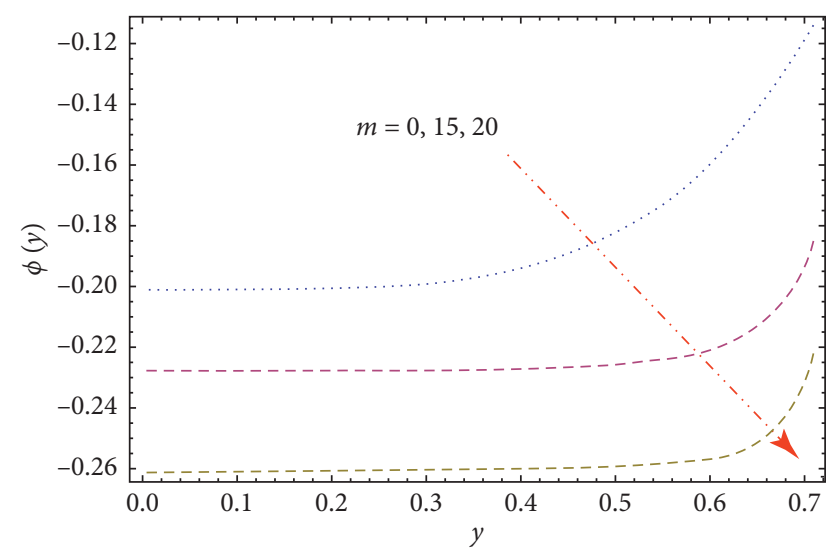

Figure 20: Graph of $\varphi(y)$ versus $m$.

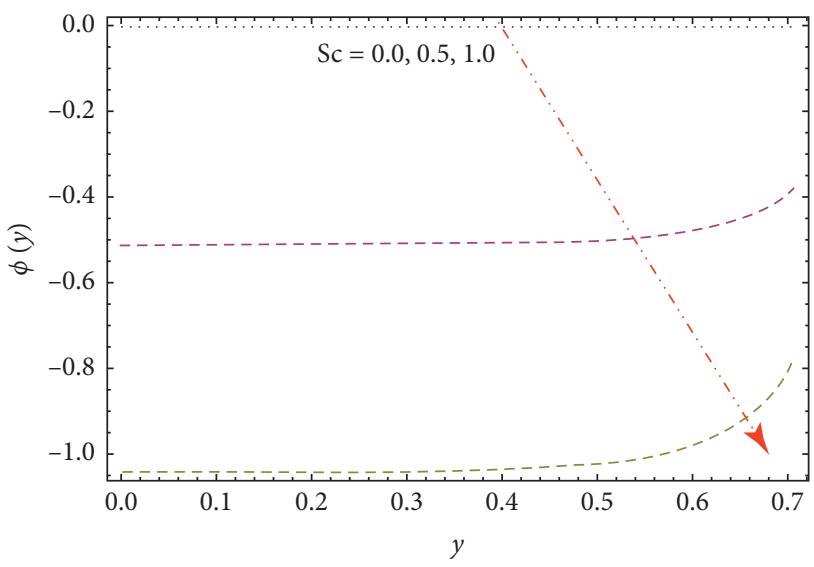

Figure 21: Graph of $\varphi(y)$ versus Sc.

improved value of the permeability parameter $k$, Figure 10 indicates an increased fluid temperature. Figure 11 shows the temperature profile difference for various electroosmotic parameter $(m)$ values. The improvement in the value of the permeability parameter increasing the fluid temperature is apparent from this figure. The fluid temperature is increased by an improvement of the value of Schmidt Sc in Figure 12. From Figure 13, it is evident that the fluid temperature is elevated with the rapid increase of the value of Soret number Sr. The effect of the Joule heating parameter $\gamma$ depicts an increase in the fluid temperature as portrayed in Figure 14. The effect of the relevant parameters on fluid concentration is reported for analysis in Figures 15-23. Figure 15 indicates that the distribution of the concentration declines with a rise in the mobility of medium $\beta$. In Figure 16, there is an upturn in the concentration profile for increasing the value of the Biot number $\mathrm{Bi}_{m}$ (mass transfer). For the Brinkman number $\mathrm{Br}$ and Dufour number Du, Figures 17 and 18 reflect the concentration distribution $\varphi(y)$. From these figures., it can be noted that the concentration distribution declines as Brinkman number Br and Dufour number Du are increased. As the value of the permeability parameter increases, Figure 19 indicates an increase in fluid concentration. Decay in the concentration profile is seen when the value of an electroosmotic parameter is increased (see Figure 20). For

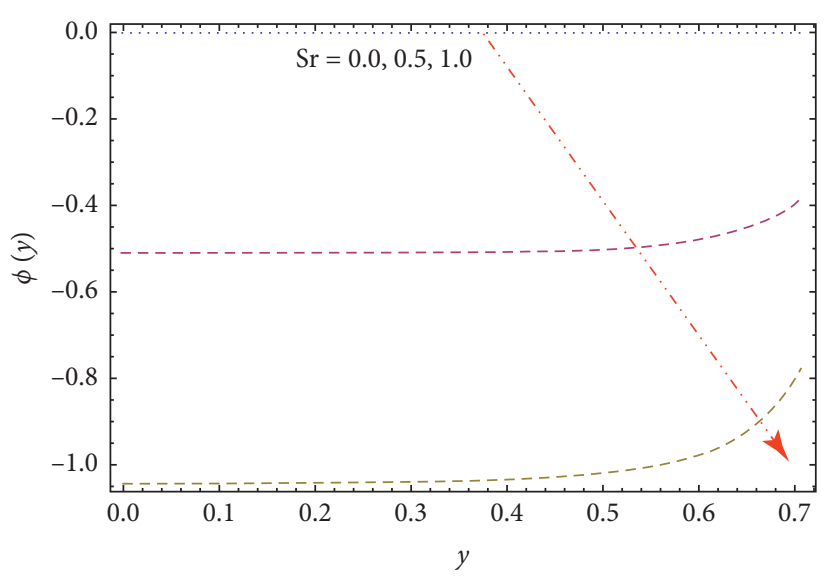

Figure 22: Graph of $\varphi(y)$ versus Sr.

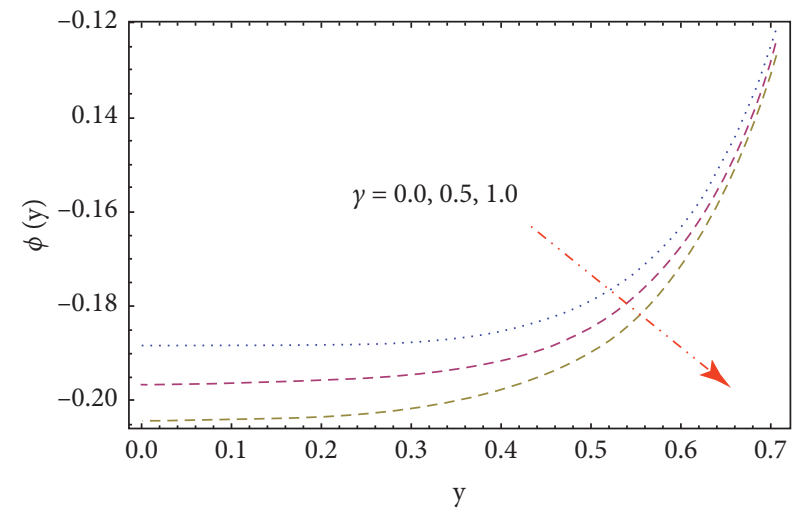

Figure 23: Graph of $\varphi(y)$ versus $\gamma$.

the Schmidt Sc and Soret Sr numbers in Figures 21 and 22, the same behavior of fluid concentration is found, meaning that the fluid concentration decays as the Schmidt or Soret numbers rise. The effect of the Joule heating parameter $\gamma$ depicts a decrease in the fluid concentration as shown in Figure 23.

\section{Concluding Remarks}

This article addresses the electroosmotic flow (EOF) with the impact of Joule heating, Soret, Dufour, and permeability in the peristaltic motion of an ionic fluid. The convective heat and mass conditions are employed at the microchannel walls. The major observations have been summarized as follows:

(i) The axial velocity is increased as the values of the electroosmotic parameter $m$ rise in the neighborhood of the channel's wall.

(ii) A boost in the value of the permeability parameter $k$ raises the fluid velocity in the middle of the channel, while the fluid speed decreases across the walls of the channel.

(iii) The axial velocity and ionic fluid temperature decay when the value of mobility of medium $\beta$ grows. 
(iv) The fluid temperature decays as the value of the thermal Biot number $\mathrm{Bi}_{m}$ is increased, whereas there is an upturn in the concentration profile for increasing the mass transfer Biot number $\mathrm{Bi}_{m}$.

(v) An increase in the temperature and concentration is noticed with a rise in the value of the permeability parameter $k$.

(vi) The fluid temperature is elevated with the rapid increase of the value of the Soret number Sr, while reverse behavior is detected for the concentration of the fluid.

(vii) In the presence of the Joule heating effect $\gamma$, the temperature and concentration distributions are reversed. Hence, it seems important not to neglect the Joule heating effect in the temperature equation to obtain more accurate results.

\section{Nomenclature}

$d: \quad$ Mean half channel width (m)

c: $\quad$ Wave speed $(\mathrm{m} / \mathrm{s})$

$\lambda: \quad$ Wavelength $(\mathrm{m})$

a: $\quad$ Wave amplitude $(\mathrm{m})$

$H, h$ : Displacement of channel walls (m)

$\bar{V}: \quad$ Velocity vector $(\mathrm{m} / \mathrm{s})$

$\vec{E}: \quad$ Applied electric field (V/s)

$\bar{u}, \bar{v}: \quad$ Velocity components $(\mathrm{m} / \mathrm{s})$

$\bar{t}: \quad$ Time $(\mathrm{s})$

$\phi$ : $\quad$ Electroosmotic potential function

$\bar{x}, \bar{y}: \quad$ Space coordinates $(m)$

$\mu$ : $\quad$ Dynamic viscosity $(\mathrm{kg} / \mathrm{ms})$

$c_{p}: \quad$ Specific heat $\left(\mathrm{m}^{2} / \mathrm{s}^{2}\right)$

$\varepsilon$ : $\quad$ Dielectric constant of the medium

$\varepsilon_{0}: \quad$ Vacuum permittivity $(\mathrm{C} / \mathrm{Vm})$

$\rho_{e}: \quad$ Density of ionic energy (ions $/ \mathrm{m}^{3}$ )

$n^{-}: \quad$ Number of anions' density

$n^{+}$: Number of cations' density

$n_{0}$ : Bulk ions concentration $\left(\mathrm{mol} / \mathrm{m}^{3}\right)$

$\kappa_{B}: \quad$ Boltzmann constant

$e: \quad$ Electric charge $(C)$

$T_{\mathrm{av}}$ : $\quad$ Average electrolyte solution temperature $(K)$

$\zeta: \quad$ Medium potential $(V)$

$\alpha: \quad$ Ionic energy parameter

$\lambda_{D}: \quad$ Debye length $(m)$

$m$ : $\quad$ Electroosmotic parameter

$k$ : $\quad$ Permeability of the medium $\left(\mathrm{N} / \mathrm{A}^{2}\right)$

$E_{x}: \quad$ Electric field along $x$-axis $(\mathrm{V} / \mathrm{m})$

$\sigma: \quad$ Electrical conductivity $(\mathrm{S} / \mathrm{m})$

$\bar{p}: \quad$ Pressure in wave frame $\left(\mathrm{N} / \mathrm{m}^{2}\right)$

$\rho: \quad$ Fluid density $\left(\mathrm{kg} / \mathrm{m}^{3}\right)$

$k_{1}$ : Thermal conductivity $(\mathrm{W} / \mathrm{km})$

$\bar{T}: \quad$ Fluid temperature $(K)$

$\bar{C}: \quad$ Fluid concentration $\left(\mathrm{mol} / \mathrm{m}^{3}\right)$

$T_{m}$ : $\quad$ Mean temperature $(K)$

D: $\quad$ Mass diffusion coefficient $\left(\mathrm{m}^{2} / \mathrm{s}\right)$

$\bar{T}_{a}, \bar{C}_{a}$ : Ambient temperature $(K)$ and concentration at walls

$h_{t}$ : $\quad$ Heat transfer coefficient $\left(W / m^{2} K\right)$ $h_{m}: \quad$ Mass transfer coefficient $\left(\mathrm{mol} / \mathrm{m}^{3}\right)$

$k_{T}$ : Thermal diffusion ratio $\left(\mathrm{m}^{2} / \mathrm{s}\right)$

$c_{s}$ : $\quad$ Susceptibility of the concentration $\left(\mathrm{m}^{3} / \mathrm{kg}\right)$

$x, y: \quad$ Dimensionless space coordinates

$u, v: \quad$ Dimensionless velocity components

$\delta: \quad$ Dimensionless wave number

$p$ : Dimensionless pressure

$\gamma: \quad$ Joule heating parameter

Pr: $\quad$ Prandtl number

Re: Reynolds number

Du: Dufour number

Br: Brinkman number

Sc: $\quad$ Schmidt number

Sr: $\quad$ Soret number

$\mathrm{Bi}_{t}$ : Heat transfer Biot number

$\mathrm{Bi}_{m}$ : Mass transfer Biot number

$\beta$ : $\quad$ Mobility of the medium

$U_{\mathrm{HS}}$ : Helmholtz-Smoluchowski velocity

$\psi: \quad$ Stream function

F: $\quad$ Dimensionless time mean flow.

\section{Data Availability}

No data were used to support this study.

\section{Conflicts of Interest}

The authors declare that they have no conflicts of interest.

\section{Acknowledgments}

This research was financially supported by the Deanship of Scientific Research, King Faisal University, Al-Ahsa, Saudi Arabia, through Nasher Track under grant no. 206135.

\section{References}

[1] N. A. Patankar and H. H. Hu, "Numerical simulation of electroosmotic flow," Analytical Chemistry, vol. 70, no. 9, pp. 1870-1881, 1998.

[2] X. Huang, M. J. Gordon, and R. N. Zare, "Current-monitoring method for measuring the electroosmotic flow rate in capillary zone electrophoresis," Analytical Chemistry, vol. 60, pp. 1837-1838, 1998.

[3] S. J. Haswell, "Development and operating characteristics of micro flow injection analysis systems based on electroosmotic flow a review," The Analyst, vol. 122, no. 1, pp. 1R-10R, 1997.

[4] P. Gravesen, J. Branebjerg, and O. S. Jensen, "Microfluidics-a review," Journal of Micromechanics and Microengineering, vol. 3, no. 4, pp. 168-182, 1993.

[5] Y. Kang, C. Yang, and X. Huang, "Electroosmotic flow in a capillary annulus with high zeta potentials," Journal of Colloid and Interface Science, vol. 253, no. 2, pp. 285-294, 2002.

[6] B. Ahmed, T. Javed, and M. Sajid, "Peristaltic transport of blood in terms of Casson fluid model through a tube under impact of magnetic field for moderate Reynolds number," Imaging Science Journal, vol. 14, pp. 101-113, 2018.

[7] B. Ahmed, T. Javed, and N. Ali, "Numerical study at moderate Reynolds number of peristaltic flow of micropolar fluid through a porous-saturated channel in magnetic field," AIP Advances, vol. 8, 2018. 
[8] B. Ahmed, T. Javed, A. H. Hamid, and M. Sajid, "Numerical analysis of mixed convective peristaltic flow in a vertical channel in presence of heat generation without using lubrication theory," Journal of Applied Fluid Mechanics, vol. 10, no. 6, pp. 1813-1827, 2017.

[9] T. W. Latham, "Fluid motion in a peristaltic pump," Master's Thesis, MIT, Cambridge, MA, USA, 1966.

[10] D. Tripathi, S. Bhushan, and O. A. Bég, "Unsteady viscous flow driven by the combined effects of peristalsis and electroosmosis," Alexandria Engineering Journal, vol. 57, no. 3, pp. 1349-1359, 2018.

[11] S. L. Weinberg, E. C. Eckstein, and A. H. Shapiro, "An experimental study of peristaltic pumping," Journal of Fluid Mechanics, vol. 49, no. 3, pp. 461-479, 1971.

[12] J. Auer and H. Krueger, "Experimental study of antiperistaltic and peristaltic motor and inhibitory phenomena," American Journal of Physiology-Legacy Content, vol. 148, no. 2, pp. 350-357, 1947.

[13] S. Na, S. Ridgeway, and L. Cao, "Theoretical and experimental study of fluid behavior of a peristaltic micropump," in Proceedings of the 15th Biennial University/Government/Industry Microelectronics Symposium, pp. 312-316, Boise, ID, USA, July 2003.

[14] X. Guo and H. Qi, "Analytical solution of electro-osmotic peristalsis of fractional Jeffreys fluid in a micro-channel," Micromachines, vol. 8, 2017.

[15] P. Jayavel, R. Jhorar, D. Tripathi, and M. N. Azese, "Electroosmotic flow of pseudoplastic nanoliquids via peristaltic pumping," Journal of the Brazilian Society of Mechanical Sciences and Engineering, vol. 41, 2019.

[16] D. Thripathi, R. Jhorar, O. A. Bég, and S. Shaw, "Electroosmosis modulated peristaltic biorheological flow through an asymmetric microchannel: mathematical model," Meccanica, vol. 53, pp. 2079-2090, 2018.

[17] N. K. Ranjit, G. C. Shit, and A. Sinha, "Transportation of ionic liquids in a porous micro-channel induced by peristaltic wave with Joule heating and wall-slip conditions," Chemical Engineering Science, vol. 171, pp. 545-557, 2017.

[18] N. K. Ranjit and G. C. Shit, "Entropy generation on electroosmotic flow pumping by a uniform peristaltic wave under magnetic environment," Energy, vol. 128, pp. 649-660, 2017.

[19] G. C. Shit, N. K. Ranjit, and A. Sinha, "Electro-magnetohydrodynamic flow of biofluid induced by peristaltic wave: a non-Newtonian model," Journal of Bionic Engineering, vol. 13, no. 3, pp. 436-448, 2016.

[20] K. Ramesh, D. Tripathi, M. M. Bhatti, and C. M. Khalique, "Electro-osmotic flow of hydromagnetic dusty viscoelastic fluids in a microchannel propagated by peristalsis," Journal of Molecular Liquids, vol. 314, Article ID 113568, 2020.

[21] A. Tanveer, S. Mahmood, T. Hayat, and A. Alsaedi, "On electroosmosis in peristaltic activity of MHD non-Newtonian fluid," Alexandria Engineering Journal, vol. 60, no. 3, pp. 3369-3377, 2021.

[22] N. Ali, S. Hussain, and K. Ullah, "Theoretical analysis of twolayered electro-osmotic peristaltic flow of FENE-P fluid in an axisymmetric tube," Physics of Fluids, vol. 32, p. 023105, 2020.

[23] S. Waheed, S. Noreen, D. Tripathi, and D. Lu, "Electrothermal transport of third-order fluids regulated by peristaltic pumping," Journal of Biological Physics, vol. 46, no. 1, pp. 45-65, 2020.

[24] S. Waheed, S. Noreen, and A. Hussanan, "Study of heat and mass transfer in electroosmotic flow of third order fluid through peristaltic microchannels," Applied Sciences, vol. 9, 2019.
[25] A. Babaie, M. H. Saidi, and A. Sadeghi, "Heat transfer characteristics of mixed electroosmotic and pressure driven flow of power-law fluids in a slit microchannel," International Journal of Thermal Sciences, vol. 53, pp. 71-79, 2012.

[26] C. H. Chen, Y. L. Hwang, and S. J. Hwang, "Non-Newtonian fluid flow and heat transfer in microchannels," Applied Mechanics and Materials, vol. 275-277, pp. 462-465, 2013.

[27] A. Sinha and G. C. Shit, "Electromagnetohydrodynamic flow of blood and heat transfer in a capillary with thermal radiation," Journal of Magnetism and Magnetic Materials, vol. 378, pp. 143-151, 2015.

[28] G. C. Shit, A. Mondal, A. Sinha, and P. K. Kundu, "Electroosmotically driven MHD flow and heat transfer in microchannel," Physica A: Statistical Mechanics and Its Applications, vol. 449, pp. 437-454, 2016.

[29] M. M. Bhatti, A. Zeeshan, R. Ellahi, and N. Ijaz, "Heat and mass transfer of two-phase flow with electric double layer effects induced due to peristaltic propulsion in the presence of transverse magnetic field," Journal of Molecular Liquids, vol. 230, pp. 237-246, 2017.

[30] N. Shehzad, A. Zeeshan, and R. Ellahi, "Electroosmotic flow of MHD power law $\mathrm{Al}_{2} \mathrm{O}_{3}$-PVC nanofluid in a horizontal channel: Couette-Poiseuille flow model," Communications in Theoretical Physics, vol. 69, 2018.

[31] A. Yadav, S. Bhushan, and D. Tripathi, "Peristaltic pumping through porous medium in presence of electric double layer," MATEC Web of Conferences, vol. 192, Article ID 02043, 2018.

[32] V. K. Narla, D. Tripathi, and G. P. R. Sekhar, "Time-dependent analysis of electroosmotic fluid flow in a microchannel," Journal of Engineering Mathematics, vol. 114, no. 1, pp. 177-196, 2019

[33] C. Yang, Y. Jian, Z. Xie, and F. Li, "Heat transfer characteristics of magnetohydrodynamic electroosmotic flow in a rectangular microchannel," European Journal of Mechanics-B/ Fluids, vol. 74, pp. 180-190, 2019.

[34] S. Hina, T. Hayat, and A. Alsaedi, "Heat and mass transfer effects on the peristaltic flow of Johnson-Segalman fluid in a curved channel with compliant walls," International Journal of Heat and Mass Transfer, vol. 55, no. 13-14, pp. 3511-3521, 2012.

[35] A. Riaz, R. Ellahi, and S. M. Sait, "Role of hybrid nanoparticles in thermal performance of peristaltic flow of eyring-powell fluid model," Journal of Thermal Analysis and Calorimetry, vol. 143, no. 2, pp. 1021-1035, 2021.

[36] T. Hayat, M. Javed, and A. A. Hendi, "Peristaltic transport of viscous fluid in a curved channel with compliant walls," International Journal of Heat and Mass Transfer, vol. 54, no. 7-8, pp. 1615-1621, 2011.

[37] O. U. Mehmood, A. A. Qureshi, H. Yasmin, and S. Uddin, "Thermo-mechanical analysis of non-Newtonian peristaltic mechanism: modified heat flux model," Physica A: Statistical Mechanics and its Applications, vol. 550, Article ID 124014, 2020.

[38] H. Yasmin, N. Iqbal, and A. Tanveer, "Engineering applications of peristaltic fluid flow with Hall current, thermal deposition and convective conditions," Mathematics, vol. 81710 pages, 2020.

[39] H. Yasmin, S. Farooq, M. Awais, A. Alsaedi, and T. Hayat, "Significance of heat and mass process in peristalsis of a rheological material," Heat Transfer Research, vol. 50, no. 16, pp. 1561-1580, 2019.

[40] H. Yasmin, N. Iqbal, and A. Hussain, "Convective heat/mass transfer analysis on Johnson-Segalman fluid in a symmetric 
curved channel with peristalsis: engineering applications," Symmetry, vol. 12, Article ID 1475, 2020.

[41] T. Hayat, A. Bibi, H. Yasmin, and F. E. Alsaadi, "Magnetic field and thermal radiation effects in peristaltic flow with heat and mass convection," Journal of Thermal Science and Engineering Applications, vol. 10, Article ID 051018, 2018.

[42] Y.-Q. Song, H. Waqas, K. Al-Khaled et al., "Bioconvection analysis for sutterby nanofluid over an axially stretched cylinder with melting heat transfer and variable thermal features: a Marangoni and solutal model," Alexandria Engineering Journal, vol. 60, no. 5, pp. 4663-4675, 2021.

[43] K. Hosseinzadeh, S. Salehi, M. R. Mardani, F. Y. Mahmoudi, M. Waqas, and D. D. Ganji, "Investigation of nano-bioconvective fluid motile microorganism and nanoparticle flow by considering MHD and thermal radiation," Informatics in Medicine Unlocked, vol. 21, Article ID 100462, 2020.

[44] H. Yasmin, T. Hayat, A. Alsaedi, and H. H. Alsulami, "Peristaltic flow of Johnson-Segalman fluid in asymmetric channel with convective boundary conditions," Applied Mathematics and Mechanics, vol. 35, no. 6, pp. 697-716, 2014.

[45] N. S. Akbar, A. B. Huda, M. B. Habib, and D. Tripathi, "Nanoparticles shape effects on peristaltic transport of nanofluids in presence of magnetohydrodynamics," Microsystem Technologies, vol. 25, no. 1, pp. 283-294, 2019.

[46] S. Noreen, Quratulain, and D. Tripathi, "Heat transfer analysis on electroosmotic flow via peristaltic pumping in non-darcy porous medium," Thermal Science and Engineering Progress, vol. 11, pp. 254-262, 2019.

[47] K. Hosseinzadeh, S. Roghan, A. R. Mogharrebi, A. Asadi, and D. D. Ganji, "Optimization of hybrid nanoparticles with mixture fluid flow in an octagonal porous medium by effect of radiation and magnetic field," Journal of Thermal Analysis and Calorimetry, vol. 143, no. 2, pp. 1413-1424, 2021.

[48] M. Khan, A. Hussain, M. Y. Malik, T. Salahuddin, and F. Khan, "Boundary layer flow of MHD tangent hyperbolic nanofluid over a stretching sheet: a numerical investigation," Results in Physics, vol. 7, pp. 2837-2844, 2017.

[49] R. Ellahi, S. M. Sait, N. Shehzad, and N. Mobin, "Numerical simulation and mathematical modeling of electro-osmotic Couette-Poiseuille flow of MHD power-law nanofluid with entropy generation," Symmetry, vol. 11, Article ID 1038, 2019.

[50] A. Riaz, S. U.-D. Khan, A. Zeeshan, S. U. Khan, M. Hassan, and T. Muhammad, "Thermal analysis of peristaltic flow of nanosized particles within a curved channel with secondorder partial slip and porous medium," Journal of Thermal Analysis and Calorimetry, vol. 143, no. 3, pp. 1997-2009, 2021.

[51] A. Riaz, A. Zeeshan, M. M. Bhatti, and R. Ellahi, "Peristaltic propulsion of Jeffrey nano-liquid and heat transfer through a symmetrical duct with moving walls in a porous medium," Physica A Stat. Mech. Appl, vol. 545, Article ID 123788, 2020.

[52] P. Goswami and S. Chakraborty, "Semi-analytical solutions for electroosmotic flows with interfacial slip in microchannels of complex cross-sectional shapes," Microfluidics and Nanofluidics, vol. 11, no. 3, pp. 255-267, 2011.

[53] D. Tripathi, S. Bhushan, and O. A. Bég, "Transverse magnetic field driven modification in unsteady peristaltic transport with electrical double layer effects," Colloids and Surfaces A: Physicochemical and Engineering Aspects, vol. 506, pp. 32-39, 2016.

[54] Y. Kikuchi, "Effect of leukocytes and platelets on blood flow through a parallel array of microchannels: micro-and macroflow relation and rheological measures of leukocyte and platelet activities," Microvascular Research, vol. 50, no. 2, pp. 288-300, 1995. 